\title{
Revisiting Temperaments with a Fine-tuned Categorization Using Iranian Traditional Medicine General Ontology
}

Ayeh Naghizadeh ${ }^{1}$, Donya Hamzehyian, Shaghayegh Akbari, Hossein Rezaeizadeh ${ }^{1}$, Mahdi Alizadeh Vaghasloo $^{1}$, Mehdi Mirzaie ${ }^{2}$, Mehrdad Karimi ${ }^{*}$, Mohieddin Jafari ${ }^{3 *}$

${ }^{1}$ Department of Persian Medicine, School of Traditional Medicine, Tehran University of Medical Sciences, Tehran, Iran

${ }^{2}$ Department of Applied Mathematics, Faculty of Mathematical Sciences, Tarbiat Modares University, Jalal Ale Ahmad Highway, Tehran, Iran.

${ }^{3}$ Research Program in Systems Oncology, Faculty of Medicine, University of Helsinki, Helsinki, Finland

*Corresponding authors:

Mohieddin Jafari, Ph.D.

Mohieddin.jafari@helsinki.fi

Mehrdad Karimi, MD, Ph.D.

mehrdadkarimi@yahoo.com

\begin{abstract}
Background: Iranian traditional medicine is a holistic school of medicine with a long prolific history. It describes numerous concepts and the relationships between them. However, no unified terminology has been proposed for the concepts of this medicine up to the present time. Considering the extensive use of concepts in the numerous textbooks written by the scholars over centuries, comprehending the totality of the terminology is obviously a very challenging task. To resolve this issue and overcome the obstacles, and code the concepts in a reusable manner, constructing an ontology of the concepts of Iranian traditional medicine seems a necessity.

Methods: Makhzan al-Advieh, an encyclopedia of materia medica compiled by Mohammad Hossein Aghili Khorasani, was selected as the resource to create an ontology of Mizaj. The steps followed to accomplish this task included (1) compiling the list of classes for Mizaj; (2) arranging the classes in taxonomy; (3) determining object properties and their cardinalities; (4) specifying annotation properties including codes, labels, synonyms, and definitions for each concept; (5) reviewing the fields pertaining to Mizaj of all monographs in Makhzan al-Advieh. The ontology was created using Protégé with adherence to the principles of ontology development provided by the Open Biological and Biomedical Ontology (OBO) foundry.

Results: Mizaj ontology was constructed with a final inclusion of 105 classes, three object properties, and 1078 axioms in the Iranian Traditional Medicine General Ontology database, IrGO, freely available at http://iafarilab.com/irgo/. An indented tree view and an interactive graph view using WebVOWL were used to visualize the ontology. All classes were linked to their instances in the UNaProd database to create a knowledge-base of Mizaj.
\end{abstract}


Conclusion: We constructed an ontology-based knowledge base of ITM concepts of Mizaj in the domain of materia medica to help offer a shared and common understanding of this concept, enable reuse of the knowledge, and make the assumptions explicit. Extending IrGO will bridge the gap between traditional and conventional schools of medicine and help guide future research on new treatment options.

Keywords: Iranian traditional medicine, Persian Medicine, ontology, knowledge-base, Mizaj, temperament, new drug discovery

\section{Introduction}

Iranian traditional medicine, also known as Persian medicine and familiar to some as Unani medicine, is a holistic school of medicine, founded on a philosophical theory with a categorical framework. The body of collected knowledge in ITM has been extensively documented and revised throughout the centuries. Persian scholars preserved a large amount of the Greco-Roman medicinal application of plants while eventually complementing and expanding it with their domestic knowledge and experiences [2]. The scientific basis of ITM, evident through many examples including Avicenna's Canon of Medicine, being a primary medical reference in Europe until the $16^{\text {th }}$ century [3], make this traditional school of medicine a powerful and innovative source for acquisition of data. Iranian scholars have used extensive terminology to describe concepts of this medical school. However, the numerous concepts are, at times, not explicit, both in terms of definition and in the complex relationships between them. Coupled with a large amount of data collected through thousands of years, this ambiguity impedes the acquisition of knowledge from this precious resource.

To address this issue and code the concepts in a reusable manner, constructing an ontology of the concepts of Iranian traditional medicine seems a necessity. An ontology is a formal explicit description of a domain, consisting of taxonomy as the backbone, universals and defined classes, and the relationships that exist among them [4]. It serves to represent the structure of a domain in order to encode specific information about the theories and preliminary principles of that science and elucidate the implicit laws. The semantic network of an ontology consists of nodes that represent entities and edges that represent the semantic relationships that exist between the concepts.

Such representation will eliminate ambiguities in concepts and provide an integrated terminology in this domain. As a result, an understanding of the natural language of ITM by intelligent systems, analysis of the relationships between concepts, and extraction of hidden relationships will be made possible. It will serve to integrate data across heterogeneous databases [5]. Moreover, the accuracy of clinical diagnosis and decision-making in treatment can be enhanced through building an ontology and knowledge-base of ITM concepts.

Over the last decades, ontologies have become prevalent in biomedicine [1]. An example in the biomedical domain includes Gene Ontology (GO) [6], used as a means to standardize terminology, to enable access to domain knowledge, to verify data consistency and to facilitate integrative analyses over heterogeneous biomedical data [5]. Moreover, there is a trend towards standardizing traditional medical systems by creating semantic networks and ontologies. One of the most comprehensive works in this regard is the traditional Chinese Medicine Language System (TCMLS), with over 120,000 concepts, 300,000 terms, and 1.27 million semantic relational links [7]. TCMLS is an extensive semantic network and thesaurus, which has recently been used to create a middle-level ontology of TCM concepts to strengthen the foundations for natural language processing, semantic retrieval, clinical decision making in traditional Chinese medicine [8]. 
No study has yet been conducted to create a language system or ontology of ITM concepts to the best of our knowledge. A major domain of any medical system is materia medica, i.e., the body of collected knowledge about the therapeutic properties of any substance used for healing. Furthermore, one of the most critical treatment principles in ITM is taking into account the Mizaj (temperament) [9] of patients, diseases, and drugs [10, 11]. Thus, creating an ontology and knowledge-base of Mizaj as the first step in formal representation of ITM concepts seems relevant. Besides aiding ITM physicians in research and decision making, it will also be an aid in new drug discovery. Utilizing the ethnomedical use of plants based on traditional medical systems is a starting point to discover new drugs $[12,13]$, which can be more effectively carried out one the information from these resources has been standardized and systematized.

This research was designed to systematically collect the concepts used to describe ITM materia medica regarding their Mizaj in order to create an ontology and subsequently, an ontologybased knowledge-base of Mizaj.

\section{Material and Methods}

\section{Domain and scope}

One of the most important domains in any medical system is the treatment modalities. In the case of traditional medicines, a chief element of treatment is the use of natural medicinal substances, including herbs, minerals, and animal-derived drugs. IrGO is intended to represent the concepts used to describe materia medica in ITM literature, with the first version (IrG0 1.0) being focused on Mizaj.

\section{Resource}

To make the ontology both comprehensive and accomplishable, a single resource was selected according to the criteria of being the most recent, containing the largest number of monographs, and being compiled by a prominent ITM scholar [14]. Makhzan al-Advieh, composed by Mohammad Hossein Aghili Khorasani, a renowned physician and pharmacologist of the $18^{\text {th }}$ century, was selected as the resource for this project. One of the best and most comprehensive resources of ITM, this semistructured encyclopedia includes 1741 principal herbal, animal, and mineral preliminary monographs. Drugs are described by their identity, Mizaj, actions and medicinal uses, dosage, adverse effects, substitutes, and refinements [15]

\section{Ontology creation}

Protégé 5.5.0, which supports the latest Web Ontology Language (OWL) specification and is the most extensively used editor for development and management of ontologies [16] was used to construct the ontology of Mizaj. The principles of ontology development provided by the OBO Foundry (http://obofoundry.github.io/principles/fp-000-summary.html) were used.

To develop the ontology, a number of steps were followed. The first task included compiling the list of classes for Mizaj. This was accomplished by text mining the description of Mizaj and the actions of monographs fields in Makhzan al-Advieh. Then, frequent terms pertaining to each of the two fields were extracted. Moreover, Avicenna's Canon of Medicine [17] was studied as one of the most essential reference books regarding the foundations of ITM that provides explanations and classifications for Mizaj concepts.

The classes were then arranged in a taxonomy, which is a hierarchy consisting of the concepts linked by sub-type relations. The selected method to construct the taxonomy was a combination of 
top-down and bottom-up approaches. An initial approach was to use a top-down method, as the more general terms are defined and already organized in descriptive form in ITM literature. However, there were also classes in lower levels which were not defined explicitly in textbooks but used to describe monographs in our selected resource. These concepts were studied, and their specifications were determined to be arranged using a bottom-up strategy to complete the model.

Each class was subsequently provided with an Aristotelian definition that takes into account the genus and differentia of terms [4]. This formulation of definitions ensures the correctness of the hierarchy, with each definition taking us back to the root node when unpacked. Moreover, circularity is automatically avoided. Root nodes were defined based on ITM literature.

The next step consisted of determining object properties and their cardinalities. Subsequently, annotation properties, including a code, a label, synonyms, and definitions, were described for each concept. Finally, the fileds pertaining to Mizaj of all monographs in Makhzan alAdvieh were reviewed manually to ensure complete extraction of all classes.

Consistency was checked using the HermiT 1.3.8.413 plugin [18]. The ontology was evaluated based on structural features and concerning its performance on text-mining tasks.

\section{Results}

\section{A review of the concept of Mizaj}

The foundational natural philosophy of ITM explains phenomena by the two pairs of opposing qualities, namely hotness-coldness and wetness-dryness. The qualities of hotness and coldness affect wetness and dryness, thus called active qualities, whereas the latter pair are affected by hotness and coldness, thus called passive qualities. The four illustrative elements of fire, wind, water, and earth that exhibit specific qualities with that of the fire being hot and dry, the wind hot and wet, the water cold and wet and the earth cold and dry. It is noteworthy that, these elements are symbolic, and not literally, what we perceive of them in the physical world. They are indivisible matter named according to the qualities and potentialities they induce in a substance. For example, the presence of fire in a substance makes it light-weighted and permeable, whereas water allows it to possess fluidity and flexibility.

The elements constitute the primary components of virtually all matter, living and non-living. When intermixed in various proportions, they act upon and react with one another, ultimately reaching a state of equilibrium and a new quality called Mizaj or temperament. As a critical concept of Iranian traditional medicine, Mizaj is an attribute of all beings in the universe. In addition to living and non-living things, a certain Mizaj is attributed to variables such as place, climate, and season, according to the prediction of the effect they have on the human body. Materia medica, whether herbal, animal, or mineral are no exception, the Mizaj of which is determined based on the change of body Mizaj in a healthy young adult upon consuming the substance.

Upon entering a balanced body and actualization of potentials under the influence of metabolic processes, a substance with a balanced Mizaj does not change the principle Mizaj of the body, nor does it lead to any deficit or disturbance in body functions. Unbalanced substances, on the other hand, can change the principle temperament of the body, leading to alterations in body functions. The magnitude of these changes is expressed as Mizaj degrees in a range from one to four for each of the qualities, each of which is further classified into three degrees of minimum, medium, and maximum. For example, a drug with a hot and dry Mizaj is described also via a degree in hotness and a degree in dryness.

Ten main Mizaj types in two major classes of balanced and unbalanced have been defined for materia medica, with the unbalanced ones as follows: hot, cold, wet, dry, hot-wet, hot-dry, cold-wet, 
cold-dry, and Morakkab al-Ghovaa (a Mizaj ascribed to drugs that can exhibit two or more of the mentioned Mizaj types).

\section{Classes in Mizaj ontology}

Text mining the Mizaj field of drugs revealed that ITM scholars used more precise terms in explaining the Mizaj of drugs. These terms mainly pertained to the degree of qualities but also resulted in the necessity to create classes other than the ten main ones (the most inner and outer circles in Figure 1) in the hierarchy of Mizaj type. There were drugs with a balanced Mizaj that were said to have slight degrees of one or two qualities. Examples include "Judas tree" (Orjovan), which is balanced though with slight hotness, and "common marshmallow" that has a balanced Mizaj with slight coldness and wetness. Therefore, eight subtypes were created for the balanced class, four with slight degrees in only one quality (demonstrated on the vertical/horizontal axis in Figure 1), and four with slight degrees in both an active and a passive quality (the green third inner circle in Figure 1).

Moreover, some drugs had slight degrees in one of the qualities, and a full degree for another. For example, "black gram" has an unbalanced cold Mizaj with a slight dryness. As these instances did not fit into either hot or hot-dry Mizaj types, eight further classes were also added to the hierarchy (the second inner circle in Figure 1).

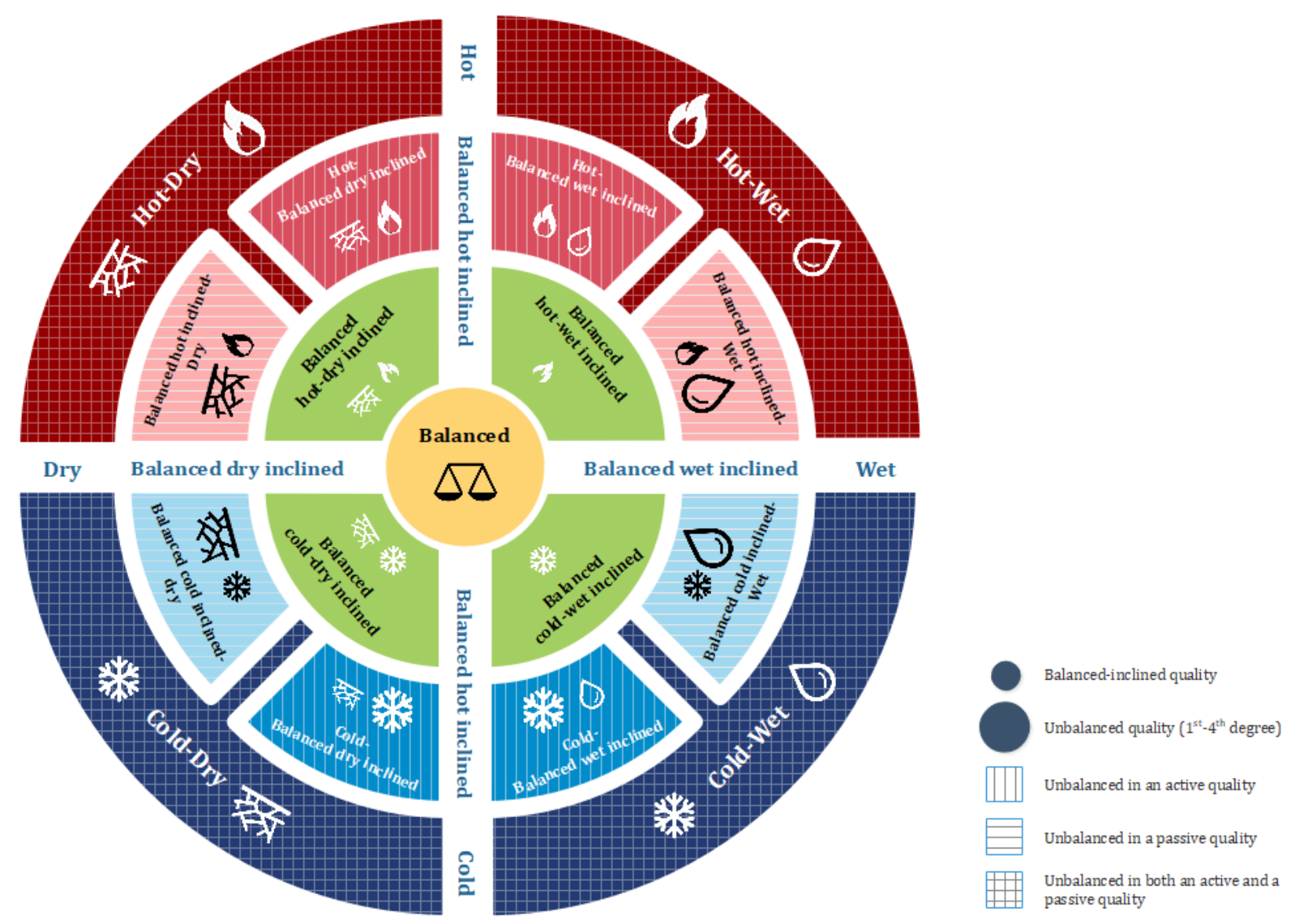

Figure 1. Mizaj types. The vertical axis is an indicator of active degrees with points lower than balanced being cold and higher points being hot. The horizontal axis displays passive degrees, with the dry degree to the left. The nine main mizaj types of ITM include balanced, hot, cold, dry, wet, hot-wet, cold-wet, hot-dry, cold-dry. Other Mizaj types illustrated in the second and third 
inner circles of the figure are more precise indications of Mizaj degrees used in descriptions of the Mizaj of drugs in Makhzan al-Advieh with the green zone in the balanced region and the light red/blue zones counting as unbalanced in one active/passive quality. Smaller icons of qualities demonstrate a balanced inclined quality while larger ones are indicative of an unbalanced quality, which can lie in a range between first to fourth degree. Mizaj types with horizontal stripes are unbalanced in one of the passive qualities, while the vertically-striped are unbalance in an active quality. The four checkered Mizaj types are unbalanced in both their active and passive quality.

As described above, the Mizaj degree is the power of the qualities to impose changes in the body. It is expressed in four degrees, where each is further classified into minimum, medium, and maximum (Figure 2). The higher the degree of a drug is, the more potent it would be in changing the Mizaj and body functions. A drug in the first degree can change the Mizaj of a person only when consumed in large amounts or over a long time but leads to no disturbance in body functions. Second degree drugs tend to change body Mizaj in small amounts, but again do not result in body dysfunctions even in large amounts or constant use. Drugs in the third degree can lead to a disturbance in body functions in addition to changing the Mizaj, while fourth degree drugs cause severe disturbance and may be lethal. Based on the terms extracted by text mining, a slight degree was also ascribed to each of the four qualities. This class indicates an inclination of a balanced Mizaj towards one of the qualities that do not reach a first degree.

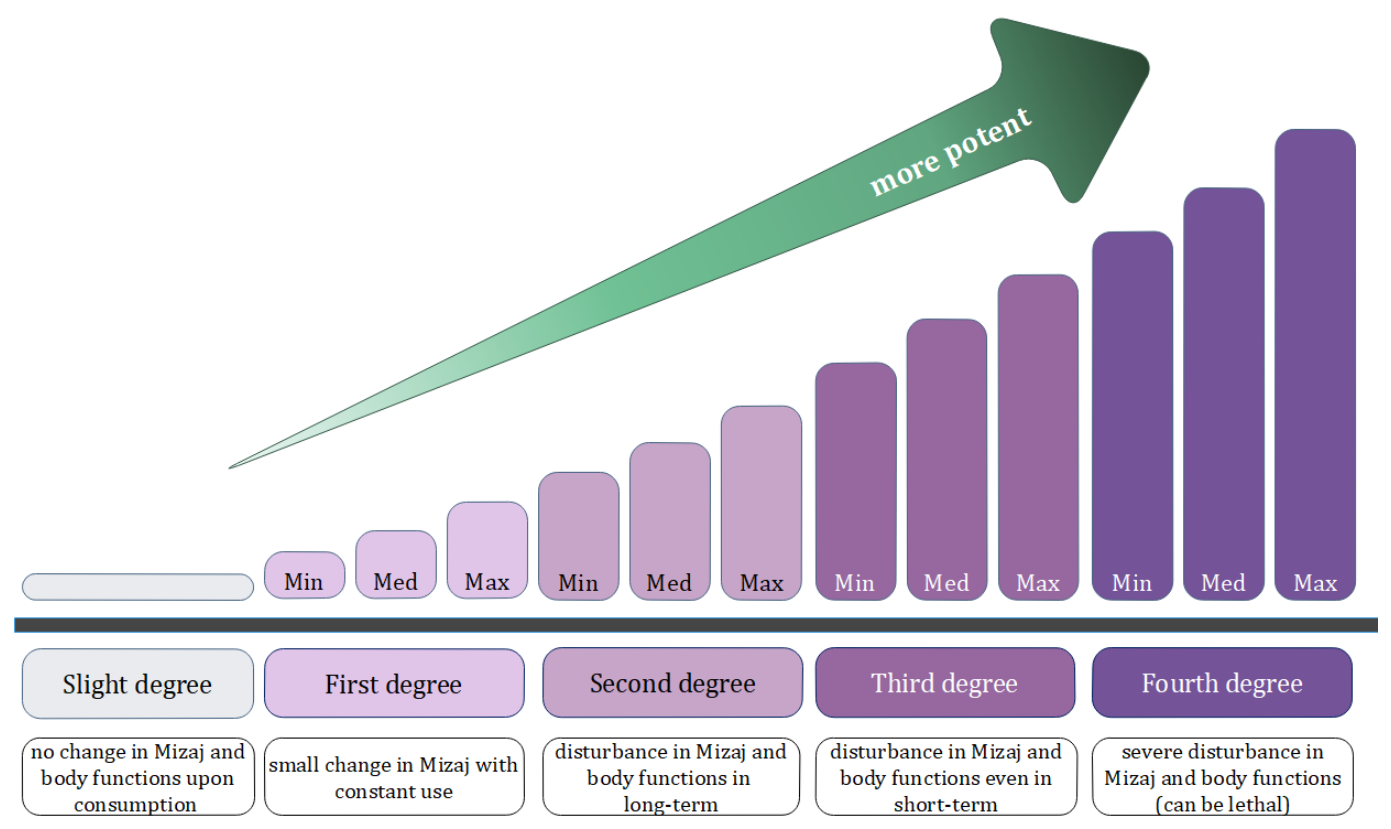

Figure 2. Mizaj degrees. The degrees used to describe each of the four qualities (hotness, coldness, wetness, dryness) range from slight to maximum in the fourth degree. This classification is according to the potency to induce changes in Mizaj and body functions of a young, healthy person upon consumption.

\section{Construction of Mizaj ontology}

Following extraction of terms for the ontology, classes were arranged in a taxonomy using a combination of top-down and bottom-up strategies and provided with definitions. Subsequently 
object properties and cardinalities were determined with a final inclusion of 105 classes, 3 object properties, and 1078 axioms. Table 1 provides a summary of ontology metrics.

Table 1. Metrics of ontology in IrGO

\begin{tabular}{|cc|}
\hline Name & Mizaj ontology (n) \\
\hline Axiom & 1078 \\
\hline Logical axiom & 343 \\
\hline Declaration axiom & 115 \\
\hline Class & 105 \\
\hline Object property & 3 \\
\hline SubClassOf & 333 \\
\hline Maximum depth & 5 \\
\hline Maximum number of children & 17 \\
\hline Annotation Assertion & 616 \\
\hline
\end{tabular}

\section{Ontology visualization}

There are a number of methods to visualize ontologies. A suitable way should support the presentation of ontology ingredients, i.e., classes, relations, instances, and properties, and allow the user to discern information without effort [19]. The Visual Notation specifies a visual language for ontologies represented in OWL for OWL Ontologies (VOWL). Two common ontology visualization techniques are the indented trees and directed acyclic graphs [20]. The indented tree was provided as an organized hierarchical view of IrGO. Also, the graph visualization, which is more controllable and intuitive was presented for the users. We created an interactive graph view using WebVOWL [21] to allow exploration and customization of ontology (Figure 3).

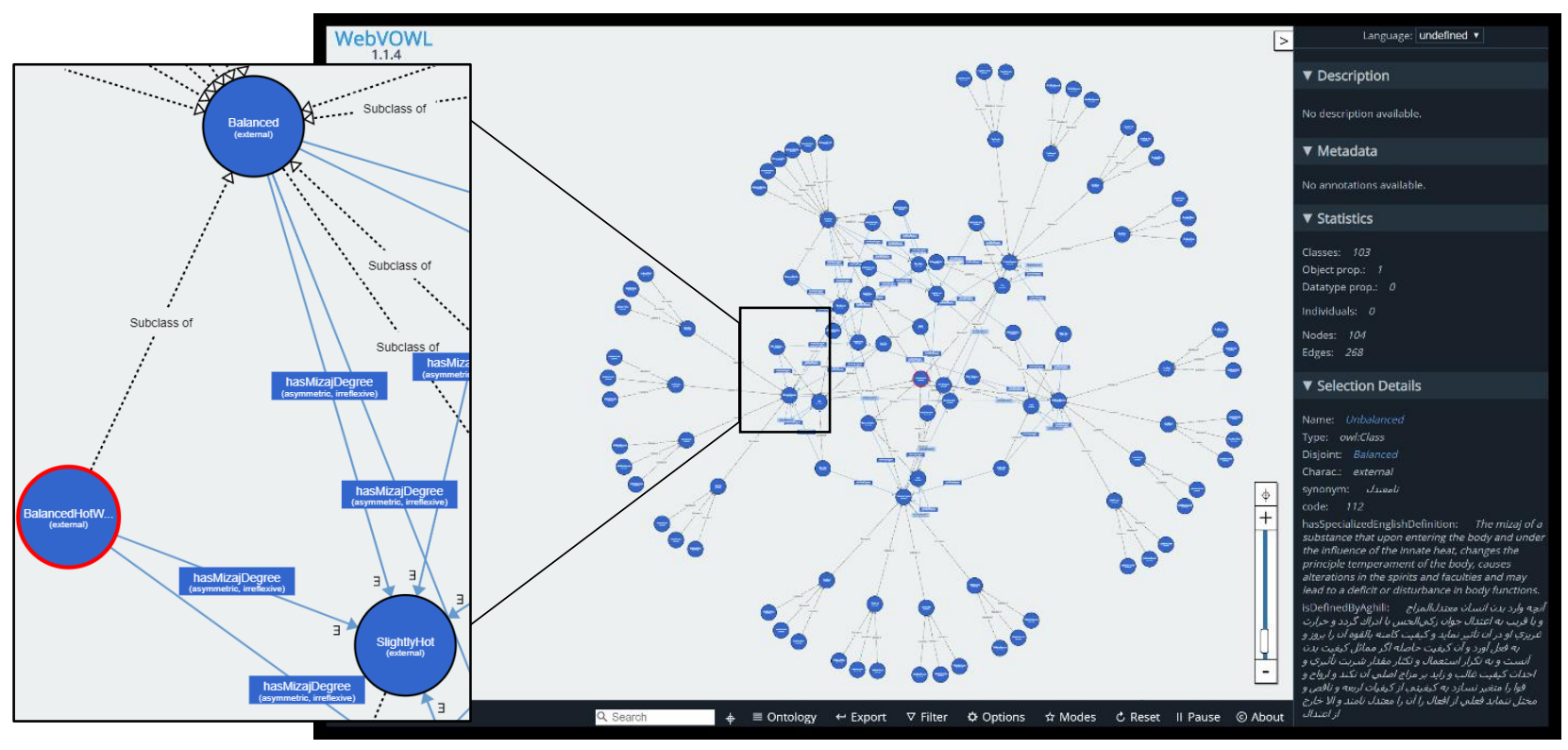

Figure 3- Network visualization of Mizaj ontology using WebVOWL. 


\section{Web Interface}

IrGO was built using PHP 7.2.11 for server-side data processing, and JavaScript ECMAScript 2015. Data was stored in MySQL 10.1.37. An outline and user manual of the database, together with network and tree views of ontology, are available on the website. The query of database contents by one or more keywords is also provided for users. Both Mizaj types and degrees are linked to their instances in UNaProd to create a knowledge-base of Mizaj [15].

\section{Conclusion}

We constructed an ontology-based knowledge base of ITM concepts of Mizaj in the domain of materia medica to standardize and clearly demarcate concepts in this regard. This was accomplished according to the principles provided by the OBO foundry using Protégé.

Systematizing ITM will help reinforce the basis for natural language processing, semantic retrieval from the rich sources of this medical school, and help physicians of this field in better clinical decision making. It also has implications in modern medical research, including ethnopharmacology and new drug discovery. Identifying potential new medicines is a process that can be fulfilled through various methods including random (biodiversity-based) [22], chemo-systematic [23], ecological [24], computational [25], and ethnopharmacological [26] approaches. Compared with the random model, utilizing ethnomedical use of plants method is time and money-saving and has a higher hitrate $[13,27]$.

Several studies have been dedicated to investigating the attributes of medicinal substances in ITM resources and current data on chemical compounds of drugs. For example, a strong relationship has been demonstrated between the Mizaj of drugs in ITM with their major chemical compounds [28]. It seems that the knowledge and experience provided by ITM can help reduce the expenses and time consumed in the process of developing new drugs from natural sources. The efficacy of this method would be even higher in a more organized and standardized model of traditional medicine. Accordingly, the ontology of Mizaj can be used to investigate similarities in chemical compounds, properties of medicinal substances, and the links to associated biological processes and diseases.

We plan to extend the ontology to include all the concepts used to describe materia medica and to create a more comprehensive knowledge-base by linking the ontologies to UNaProd, a database of ITM materia medica. In conclusion, developing an ontology-based knowledge-base of ITM concepts will provide a whole range of benefits, including offering a shared and common understanding of ITM, enabling reuse of the knowledge in this field, making the assumptions explicit and finally gaining new knowledge by analyzing the concepts and their relationships. It will also bridge the gap between traditional and conventional schools of medicine, which will, in turn, help guide future research on novel treatment options based on the obtained knowledge.

\section{References}

1. Nickchi, P., M. Jafari, and S. Kalantari, PEIMAN 1.0: Post-translational modification Enrichment, Integration and Matching ANalysis. Database, 2015. 2015.

2. Cragg, G.M. and D.J. Newman, Natural products: $A$ continuing source of novel drug leads. Biochimica et Biophysica Acta (BBA) - General Subjects, 2013. 1830(6): p. 3670-3695.

3. Zargaran, A., et al., Avicenna (980-1037 AD). J Neurol, 2012. 259(2): p. 389-90. 
4. Arp, R., B. Smith, and A.D. Spear, Building ontologies with basic formal ontology. 2015, Cambridge, Massachusetts [etc.]: MIT Press.

5. Hoehndorf, R., M. Dumontier, and G.V. Gkoutos, Evaluation of research in biomedical ontologies. Brief Bioinform, 2013. 14(6): p. 696-712.

6. Ashburner, M., et al., Gene ontology: tool for the unification of biology. The Gene Ontology Consortium. Nat Genet, 2000. 25(1): p. 25-9.

7. Yu, T., M. Cui, and L. Jia. Towards a TCM domain ontology: Standardization, ontology engineering, and applications. in 2015 8th International Conference on Biomedical Engineering and Informatics (BMEI). 2015.

8. Hai Long, Y.Z., Lirong Jia, Bo Gao, Jing Liu, Lihong Liu, Heinrich Herre, An ontological framework for the formalization, organization and usage of TCM-Knowledge. BMC Med Inform Decis Mak, 2019. 19(Suppl 2): p. 53.

9. Laila Shirbeigi, A.Z., Ayeh Naghizadeh, Mahdi Alizadeh Vaghasloo, The Concept of Temperaments in Traditional Persian Medicine. Traditional and Integrative Medicine, 2017. 2(3): p. 143-156.

10. Jafari, M., et al., Proteomics and Traditional Medicine: New Aspect in Explanation of Temperaments. Complementary Medicine Research, 2014. 21(4): p. 250-253.

11. Rezadoost, H., M. Karimi, and M. Jafari, Proteomics of hot-wet and cold-dry temperaments proposed in Iranian traditional medicine: a Network-based Study. Scientific Reports, 2016. 6(1): p. 30133.

12. Fabricant, D.S. and N.R. Farnsworth, The value of plants used in traditional medicine for drug discovery. Environ Health Perspect, 2001. 109 Suppl 1: p. 69-75.

13. Pan, S.-Y., et al., New Perspectives on How to Discover Drugs from Herbal Medicines: CAM's Outstanding Contribution to Modern Therapeutics. Evidence-based complementary and alternative medicine : eCAM, 2013. 2013: p. 627375-627375.

14. Modabberi, M., Aghili Alavi Khorasani and Makhzan Al-Adviyeh. Journal of The Faculty of Letters and Humanities (Kerman), 2002. Winter - Spring (8-9 (7)): p. -.

15. Naghizadeh, A.H., D.; Aldahdooh, J.; Akbari, S.; Mohammadi, F.; Otoufat, T.; Asgari, S.; Zarei, A.; Noroozi, S.; Nasiri, N.; Salamat, M.; Karbalaei, R.; Mirzaie, M.; Rezaeizadeh, H.; Tang, J.; Karimi, M.; Jafari, M., UNaProd: A Universal Natural Product Database for Materia Medica of Iranian Traditional Medicine. . Preprints, 2019050091 (doi: 10.20944/preprints201905.0091.v1), 2019.

16. Musen, M.A. and T. Protégé, The Protégé Project: A Look Back and a Look Forward. Al matters, 2015. 1(4): p. 4-12.

17. Avicenna, Qanun Fi al-Teb (Canon of Medicine). 2005, Beirut, Lebanon: Dare Ehya al-Toras Institute.

18. Motik, B., R. Shearer, and I.J.J.o.A.I.R. Horrocks, Hypertableau reasoning for description logics. 2009. 36: p. 165-228.

19. Katifori, A., et al., Ontology visualization methods\&mdash;a survey \%J ACM Comput. Surv. 2007. 39(4): p. 10.

20. Fu, B., N.F. Noy, and M.-A. Storey. Indented Tree or Graph? A Usability Study of Ontology Visualization Techniques in the Context of Class Mapping Evaluation. 2013. Berlin, Heidelberg: Springer Berlin Heidelberg.

21. Lohmann, S., et al., Visualizing Ontologies with VOWL. Semantic Web, 2016. 7(4): p. 399-419.

22. Shaneyfelt, M.E., et al., Natural products that reduce rotavirus infectivity identified by a cell-based moderate-throughput screening assay. Virology Journal, 2006. 3(1): p. 68.

23. Cook, D., et al., Detection of toxic monofluoroacetate in Palicourea species. Toxicon, 2014. 80: $\mathrm{p}$. 9-16.

24. Obbo, C.J.D., et al., Antiprotozoal activity of Khaya anthotheca, (Welv.) C.D.C. a plant used by chimpanzees for self-medication. Journal of Ethnopharmacology, 2013. 147(1): p. 220-223. 
25. Basith, S., et al., Expediting the Design, Discovery and Development of Anticancer Drugs using Computational Approaches. Curr Med Chem, 2017. 24(42): p. 4753-4778.

26. Fakhrudin, N., et al., Identification of plumericin as a potent new inhibitor of the NF-KB pathway with anti-inflammatory activity in vitro and in vivo. British Journal of Pharmacology, 2014. 171(7): p. 1676-1686.

27. Gyllenhaal, C., et al., Ethnobotanical approach versus random approach in the search for new bioactive compounds: Support of a hypothesis. Pharmaceutical Biology, 2012. 50(1): p. 30-41.

28. Ardekani, M.R., et al., Relationship between temperaments of medicinal plants and their major chemical compounds. J Tradit Chin Med, 2011. 31(1): p. 27-31. 